\begin{tabular}{ccc}
\hline & International Journal of Engineering \& Technology, $7(2.12)(2018) 110-114$ \\
SPC & International Journal of Engineering \& Technology \\
Website: $w w w . s c i e n c e p u b c o . c o m / i n d e x . p h p / I J E T$ & Research Paper \\
\hline
\end{tabular}

\title{
A study on the asset smart management system based on IOT
}

\author{
Moonhee Han ${ }^{1}$, Yousin Kim ${ }^{1}$, HarkSoo Park ${ }^{1}$, Dae Ho Kim ${ }^{2}$ \\ ${ }^{1}$ Dept. of Information Security KISTI, Korea \\ ${ }^{2}$ Div. of Service Management, Mokwon University, Korea \\ *Corresponding author E-mail: mhhan@kisti.re.kr
}

\begin{abstract}
Background/Objectives: Most companies use a variety of assets for their operations. However, most companies use inefficient methods, such as on-the-spot inspections once or twice a year, instead of using the newest IT technology to manage their valuable assets. Furthermore, there is no pre-control and post-management system to prevent external leakage and the loss of expensive and portable assets.

Methods/Statistical analysis: This study aims to study a practical and smart asset management system that can manage the real-time locations and the movement statuses of assets using the existing Radio-Frequency Identification (RFID) technology and the newest Bluetooth Low Energy (BLE) technology.

Improvements/Applications: Through the Smart Asset Management System developed through this study, it was possible to improve the accuracy of assets due diligence and shorten the time, and provide asset position information to the manager in real time, thereby reducing the manpower, time and cost for asset management work. As a result of the study, a Smart Asset Management System (SAMS) was developed using two IoT technologies and the system was used for 11 months in the field. When the relevant system was applied to the field, there were some effects such as, the improvement in accuracy, the reduction in input manpower, the reduction in input time, and the reduction in the number of times of surveyscompared to past manual asset management.
\end{abstract}

Keywords: IoT; Internet of Things; Asset Management System; BLE; Bluetooth Low Energy.

\section{Introduction}

Currently, the National Research Council of Science \& Technology (NST) and 25 affiliated research institutes have a large number of research assets allowing for the performance of researchers' unique missions. However, 20 of the 25 research institutes manage research assets using inefficient methods, such as on-the-spot inspections once or twice, utilizingbar code technology instead of the newest IT technology, and have no advanced prevention or postmanagement system in relation to the loss of research assets. The remaining five research institutes are using RFID technology to manage their assets and conduct due diligence. However, since they use 5 6 years old RFID technology and equipment that have problems of short distances of asset recognition and low recognition rates, they have difficulties in efficiently managing assets as they actually exist.

With a view to improving the inefficient asset management method, this study aims to develop a practical system that can simultaneously utilize the RFID technology and the BLE technology, which are two types of IoT technology. This study is based on the case of the construction of an asset management system of the governmentfunded "K" research institute affiliated to the National Science and Technology Council of Korea (NST).

\section{Related researches}

\subsection{Internet of things (IoT)}

Internet of Things (abbreviation, IoT) is a technology that embeds sensors and communication functions in items, such as various embedded systems that are included inhome appliances, mobile equipment, and wearable computers allowing those items to connect to the Internet 1, 2, 3 .

According to a survey by Gartner (2009) and Cisco Systems, "the number of IoT things will exponentially increase to 26 billion by 2020 " 4, and "the economic value during 2013-2022 will reach 14.4 trillion dollars" 5.

The technologies included in IoT are summarized as shown in Figure 16 . 


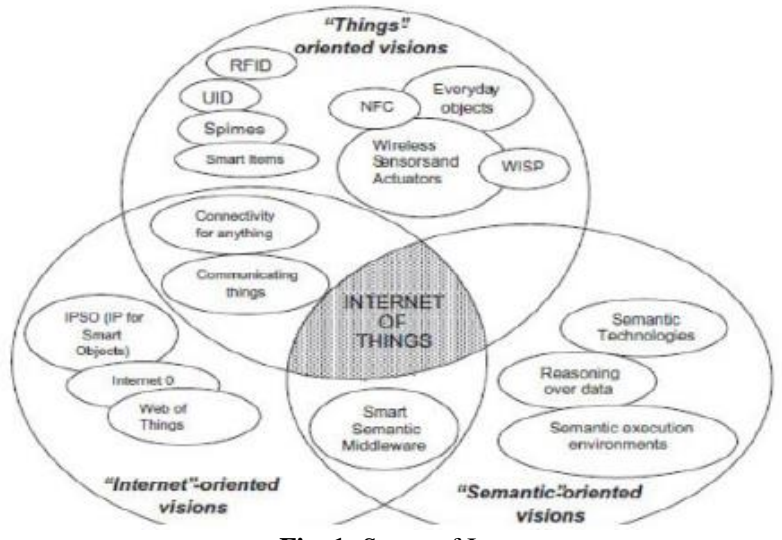

Fig. 1: Scope of Iot.

\subsection{Radio-frequency identification (RFID)}

The Radio-Frequency Identification (RFID) technology refers to technology that uses radio waves to recognize information over a long distance. An RFID system is a system that identifies IDs using frequencies. It is also called electronic tagging and consists of an RFID tag and anRFID reader. An RFID tag is composed of an antenna and an integrated circuit. The integrated circuit has a function to record information, and the antenna has a function to transmit the information to the RFID Reader. The RFID systems recognizes RFID tags through radio waves, can read tags from a long distance, and can receive information without concern for objects passing between the tag and the reader. RFID systems have many advantages and disadvantages. The advantages include the fact that they can be semi-permanently used, easy data storage, the fact that RFID tags can be recognized during movements, the fact that RFID tags can be recognized without contact, the fact that they can be repeatedly reused, and the fact that information from multiple tags can be recognized simultaneously.The disadvantages include high prices, the fact that frequencies differ country-by-country, limited ranges of the application of radio waves, and the fact that information can be leaked7. According to IDTechEx (2017), the value of the RFID market is expected to rise to US \$ 18.68 billion by 20268 .

\subsection{Bluetooth low energy (BLE)}

Beacon was originally a term that referred to devices that periodically transmit signals to inform the locations of signal fires and lighthouses and deliver various kinds of information. For beacons to play the role of reference points to inform locations and actually deliver information, communication technologies such as ultrasonic waves, infrared rays, and Bluetooth are necessary. Depending on the kinds of signals transmitted, beacons can be classified into beacons using ultrasonic waves or visible light, sound-based low-frequency beacons, LED beacons, Wi-Fi beacons, and Bluetooth beacons. In recent years, Bluetooth Low Energy (BLE) -based shortrange wireless communication devices are generally called beacons9.

BLE Beacons have advantages that includelow battery consumption compared to existing technologies, so that a BLE Beacon can be used for about two years with a small coin battery 10 and that their recognition distance is longer than of Near Filed Communication (NFC) technology, which is one of the RFID technologies, and can communicate up to around $50 \mathrm{~m}$. Because of the advantages of BLE Beacons, such as low power, low cost, and the fact that they can be applied to diverse areas of utilization, indoor location services using Beacons has been attracting attention recently11.

\section{The asset management system based on IOT}

\subsection{System structure}

The Smart Asset Management System (SAMS) has been developed to simultaneously use the Radio-Frequency Identification (RFID) technology, which was widely used in the past among IoT technologies, and the Bluetooth Low Energy (BLE) technology, which was introduced recently, using the Oracle DB to manage collected data, and WEB technology to provide user interfaces.

In this study, to develop asset management functions utilizing RFID technologies, 20,000 passive RFID tags, two RFID readers, two RFID gates, two RFID tag printers, and two notebooks were used. The RFID Tag Printers were used to input major asset information such as asset name, asset number, the date of first introduction, introduction price, and first introducer into RFID Tags in the form of stickers. The RFID Tags were used in the form of stickers and attached to assets that are not frequently moved such as desktop PCs, monitors, desks, chairs, and notebooks. The RFID tags used in this study has a size of $95 \mathrm{~mm} * 25 \mathrm{~mm} * 0.5 \mathrm{~mm}$ as shown in Figure 2.The RFID Tags were used for data communication with the RFID Readers and the $900 \mathrm{MHz}$ system, which is the standard RFID frequency band of South Korea, was used as a communication method. As shown in Figure 3, the RFID readers have a 400mm long scoop shapeand can recognize multiple RFID tags within $10 \mathrm{~m}$ simultaneously.

The RFID Gates are stand-shaped RFID readers as shown in Figure 4 and have a size of $1500 \mathrm{~mm} * 500 \mathrm{~mm} * 100 \mathrm{~mm}$. The RFID Gates were installed at the entrances and exits of buildings and were used to automatically identify the entry and exit details of the assets with attached RFID Tags. The RFID Gates utilize a middleware server connected through LAN cables to transmit the entry and exit information of the assets with attached RFID Tags to the DB Server in real time.

The notebooks were used to automatically collect the RFID tag information collected by the RFID readers and to drive the Web program with a function to transmit the collected information to the DB server in real time.

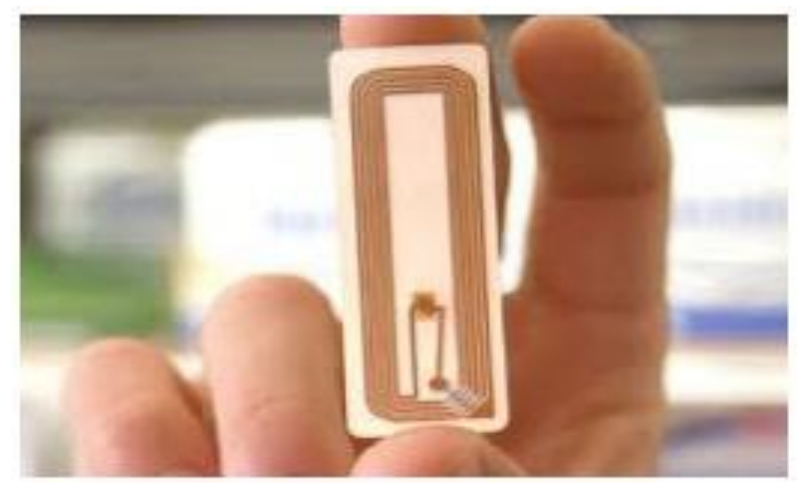

Fig. 2: Rfid Tag. 


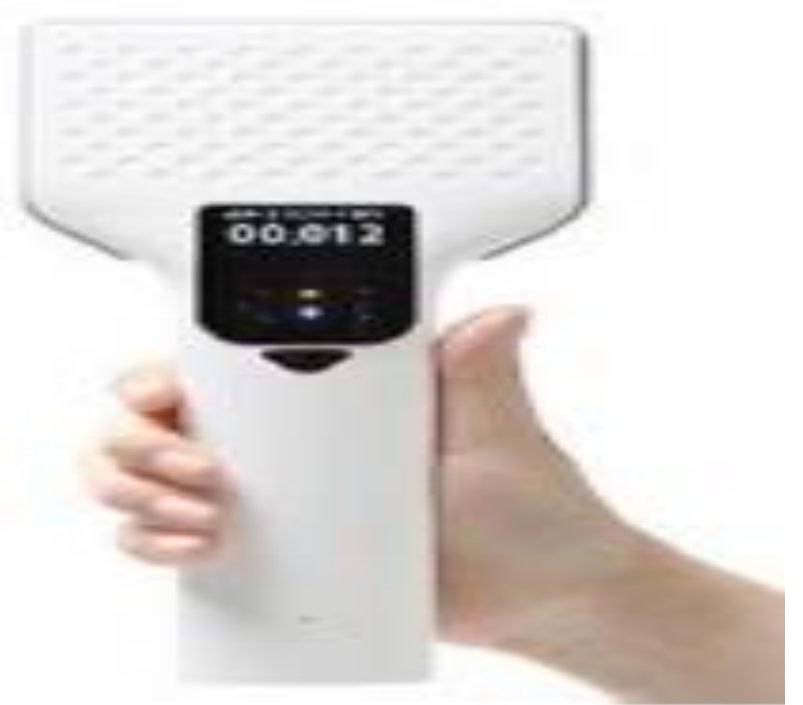

Fig. 3:.Rfid Reader.
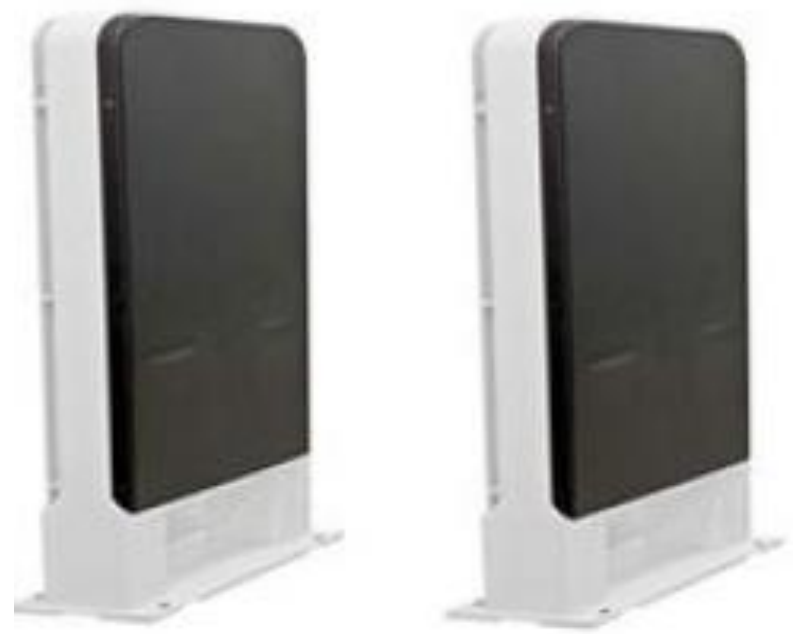

Fig. 4: Rfid Gate.

Todevelop asset management functions using the BLE technology, 50 BLE tags, four BLE Wifi /APs, three Wifi APs, and one Desktop PC were used.

The BLE Tags are $40 \mathrm{~mm} * 31 \mathrm{~mm} * 10 \mathrm{~mm}$ sized devices that transmit short distance signals as shown in Figure 5. Each of the BLE Tag has a built-in coin-shaped battery that can be used for about one year. Each Tagfunctions as a motion sensor anda buzzer (beep sound generation) as well asfor a blinking LED and allows for mutual communication with BLE Wifi / Aps in the Bluetooth 4.0 system. In this study, BLE tags were attached to 200 assets to manage high-priced assets that are frequently moved such as notebooks and tablet PCs.

The BLE Wifi/APs are sized $90 \mathrm{~mm} * 128 \mathrm{~mm} * 18.5 \mathrm{~mm}$ as shown in Figure 6 below and were used to collect information from BLE Tags within a radius of $25 \mathrm{~m}$ and transmit the collected information with to the DB Server through Wifi /APs in Figure 7.

The asset management functions using the BLE technology do not require firsthand visits to the site, unlike the RFID technology that requires firsthand visits to the site for assets due diligence and enable asset management in real time at the office using the Web program.

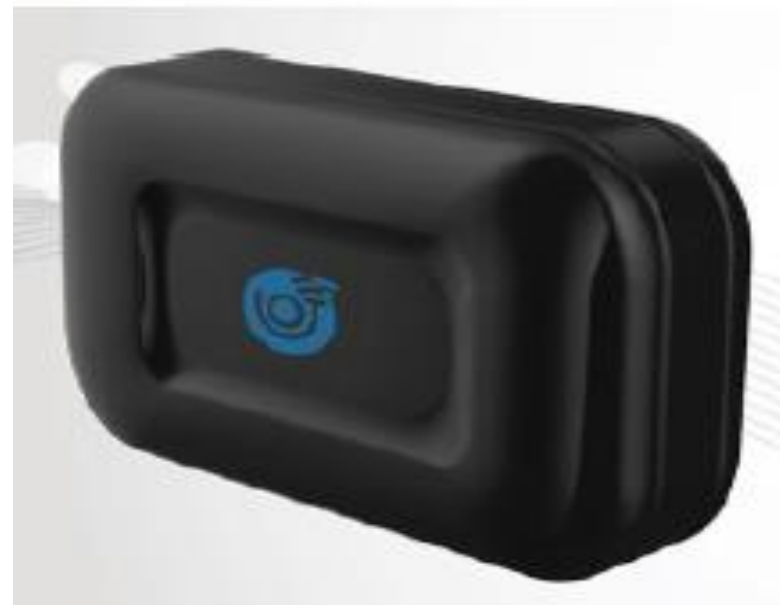

Fig. 5: Ble Tag.

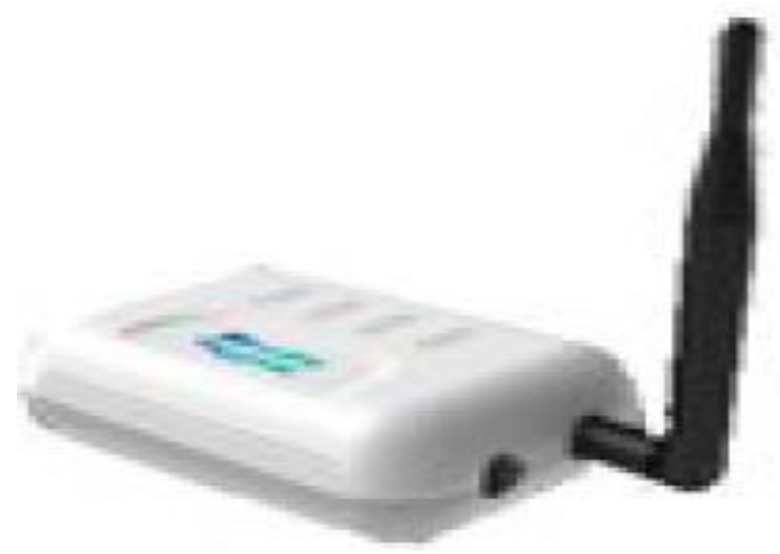

Fig. 6: Ble Wifi/Ap.

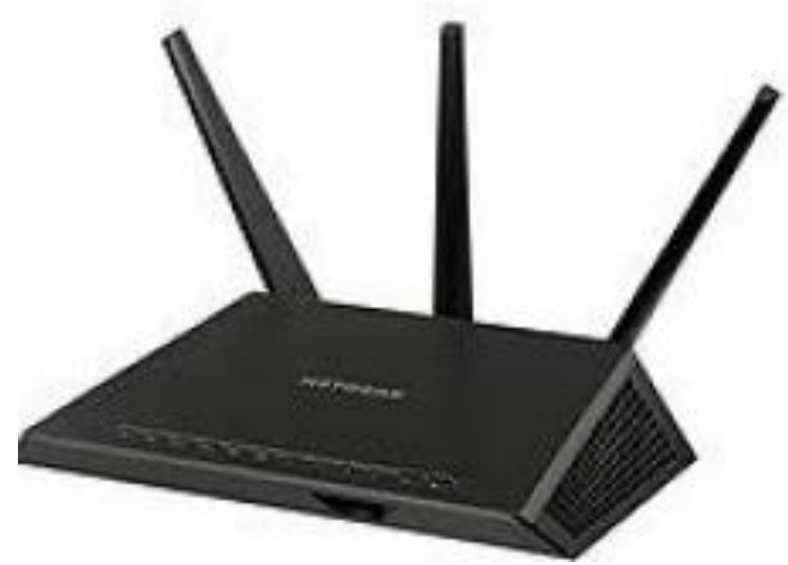

Fig. 7: Wifi/AP.

The SAMS can selectively use the RFID technology and the BLE technology according to the types of assets and the purposes of asset management and can provide asset information such as the locations of assets and details of movements in real time based on the collected data utilizing the web program. The structure of the SAMS is as shown in Figure 8 below. 


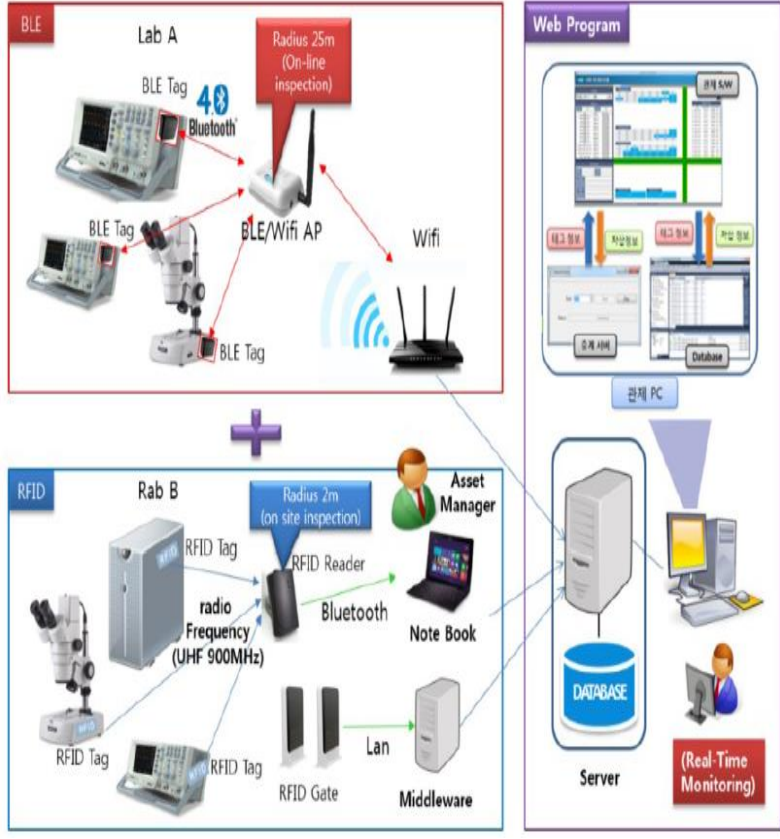

Fig. 8: The System Structure of Smart Asset Management System.

\subsection{Web service menu}

The web program for asset management has diverse functions and the main functions are basic information management, assets due diligence inspection, asset change management, and tag management.

The entire menu structure consists of five large menus and 21 small menus. The detailed menu structure is shown in Figure 9.

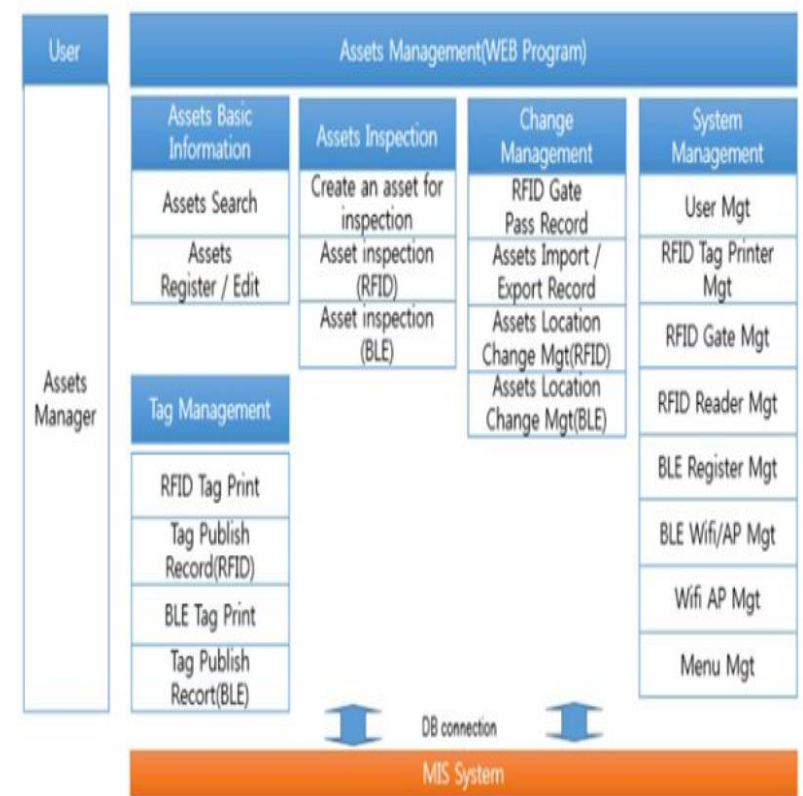

Fig. 9: Web Program Menu Configuration of Smart Asset Management System.

\section{Effect of smart asset management system}

In this study, an asset management system that can simultaneouslyuse the RFID technology and the BLE technology was developed and the developed system was actually applied to the governmentfunded ' $\mathrm{K}$ ' researchinstitution and operated for 11 monthsas shown in Table 1.

To manage assets that are not frequently moved such as desktop PCs, Monitors, notebooks, desks, and chairs among the research assets held by ' $K$ ' research institute, 20,000 RFID tags, two RFID readers, and two RFID gateways were utilized and to manage notebooks and tablet PCs that are frequently moved, 50 BLE tags, four BLE Wifi /APs, and three Wifi APs were utilized to conduct actual asset management.

During the actual 11 months of operation, many problems that had occurred in asset location identifying surveys conducted manually in the past could be solved.In particular, when the RFID technology was utilized to locate assets, unlike the assets due diligence conducted in the past, in which assets were visually inspected one by one, the locations of multiple assets at 1 2 $\mathrm{m}$ distances from each other could be accurately identified simultaneously through RFID readers, and asset entry and exit information could be managed through RFID Gates. When the BLE technology was utilized to locate assets, the locations of all assets attached with BLE tags within a radius of 20 meters from each BLE Wifi/AP could be identified in real time.

In the past, to identify the locations of assets, 'K' research institute carried out manual assets due diligence twice per year for three months, each time with three people. However, there were difficulties in accurate due diligence because of omission and duplications. When the smart asset management system was utilized, assets due diligence could be conducted more accurately than in the past through one field survey per year for one month, with two people and asset information could be monitored in real time through the web program.

Table 1: Comparison of Manpower Input

\begin{tabular}{lllll}
\hline & Before & After & $\begin{array}{l}\text { In- } \\
\text { crease }\end{array}$ & $\begin{array}{l}\text { Efficiency } \\
(\%)\end{array}$ \\
\hline $\begin{array}{l}\text { Manpower } \\
\begin{array}{l}\text { Inspection term } \\
\text { (Month) }\end{array}\end{array}$ & 3 & 2 & -1 & $33 \% \uparrow$ \\
$\begin{array}{l}\text { Count of inspection } \\
\text { (per year) }\end{array}$ & 2 & 1 & -1 & $50 \% \uparrow$ \\
$\begin{array}{l}\text { Total } \\
\text { (Man/Month) }\end{array}$ & 2 & 1 & -1 & $50 \% \uparrow$ \\
& $12 \mathrm{M} / \mathrm{M}$ & $2 \mathrm{M} / \mathrm{M}$ & - & $10 \mathrm{M} / \mathrm{M}$ \\
\hline
\end{tabular}

\section{Discussion}

In this study, a Smart Asset Management System (SAMS) was developed using two IoT technologies and the system was used for 11 months in the field. When the relevant system was applied to the field, there were effects such as,the improvement in accuracy, the reduction in input manpower, the reduction in input time, and the reduction in the number of times of surveys were conducted ascompared to past manual asset management. However, some problems such as short recognition distances and low recognition rates were found while the system was actually operated.

The biggest problems of the RFID technology wereshort recognition distances and low recognition rates of RFID tags. Although the theoretical recognition distance of RFID tags is approximately $10 \mathrm{~m}$, the stable recognition distances were shown to be $1 \sim 2 \mathrm{~m}$ at sites where there were obstacles, such as partitions, desks, chairs, and monitors. In the case of RFID gates, the recognition rates significantly decreased in cases where the RFID tags were attached to metal surfaces or covered by metal or thick books and cases where RFID tags could not be recognized. The recognition rates were improved when a $1 \mathrm{~mm}$ thick rubber shielding material was used before RFID Tags were attached to the surface of notebooks so that RFID Tags would not come into direct contact with metal. However, a disadvantage is that the thickness of the RFID tag increased occurred. If RFID Tags are developed to have a shielding function and to remain thin, the rate of use in the field will be increased.

The biggest problems of the BLE technology include the large size $(40 \mathrm{~mm} * 31 \mathrm{~mm} * 10 \mathrm{~mm})$ of BLE Tags, the short battery life (1 year), the high cost (about U\$ 8 each), and the fact that locations cannot be more accuratelylocated to than within 1 2 meters. The BLE tag size is expected to be improved thanks to the development of integration technology. The battery lifetime is expected to be improved to at least two times the current lifetime if the function of BLE Tags is improved, so that the BLE Tags operate only when called or when 
an event has occurred, instead of constantly using the power source. In addition, the problem in identifying locations is expected to be partially solved if absolute position BLE tags are attached to the office wall or column to indicate the three-dimensional coordinates (XYZ). Furthermore, it is expected that more accurate locations can be identified by attaching more absolute position BLE tags. However, if many BLE Tags, which are about U\$ 8 each, are used, a large budget is expected to be consumed for installation and maintenance.

\section{References}

[1] Brown, Eric. Who Needs the Internet of Things? https://www.linux.com/news/who-needs-internet-things, Date accessed 11/30/2017.

[2] Brown, Eric. 21 Open Source Projects for IoT. https://www.linux.com/news/21-open-source-projects-IoT. Date accessed 11/30/2017.

[3] Internet of Things Global Standards Initiative http://www.itu.int/en/ITU-T/gsi/iot/Pages/default.aspx. Date accessed 11/30/2017.

[4] Nordrum, Amy. Popular Internet of Things Forecast of 50 Billion Devices by 2020 Is Outdated. https://spectrum.ieee.org/tech-talk/telecom/internet/popular-internet-of-things-forecast-of-50-billion-devices-by-2020-is-outdated.Date accessed 11/30/2017.

[5] https://en.wikipedia.org/wiki/Internet_of_things. Date accessed $11 / 30 / 2017$

[6] L. Atzori, A. Iera, and G. Morabito, The Internet of Things: A survey.Computer Networks. 2010, 54,pp. 2787-2805

[7] https://ko.wikipedia.org/wiki/RFID. Date accessed 11/30/2017.

[8] RFID Forecasts, Players and Opportunities 2017 2027.https://www.prnewswire.com/news-releases/rfid-forecastsplayers-and-opportunities-2017-2027-300504326.html. Date accessed 11/30/2017

[9] Jung-A Lee. "IT-based Studies on Foundation of Future Society".NIA, IT \& Future Strategy Report No.8, 2014.

[10] Nam, ChoonSung; Jung, HyunHee; and Shin, DongRyeol.The Loss Prevention System of Smart Device Using by iBeacon. Journal of Internet Computing and Services. 201415 (6), pp. 27-34.

[11] Cho, Byungho, Design of Restaurant Advertisement and Order System using Bluetooth 4.0 Technology. The Journal of the Institute of Internet, Broadcasting and Communication. 2015, 15 (1), pp.69-76 